\title{
Sur la viviparité possible chez le sarcopte de la gale humaine
}

\author{
par Virgil NITZULESCU
}

Rue Sagetei 6, Bucarest, Roumanie

\begin{abstract}
Résumé
Description, dans un cas de gale norvégienne, d'un Sarcopte femelle portant à son intérieur une larve libre et entièrement formée. Pour l'auteur, la gale commune et la gale norvégienne ont le même agent étiologique. La viviparité exceptionnelle observée ici est attribuée à une hyperfertilité de cet Acare, favorisée par les conditions du terrain.
\end{abstract}

\section{Summary}

Description, in a case of Norvegian itch, of a Sarcoptes female bearing in its body a free larva. For the author, common human itch and Norvegian itch have the same etiologic agent. The exceptional viviparity of the Acarian female may be due to an hyperfertility conditioned by its localizations in the Norvegian itch.

Dans son manuel de zoologie médicale et agricole de 1895, A. Raillet décrit le sarcopte femelle de la gale humaine comme: «ovipare, quelquefois vivipare, d'après Bourguignon $» . .$. (page 647).

Nous n'avons pas pu trouver le texte original de Bourguignon, et il ne nous est pas très clair ce que veut dire ici le mot: «quelquefois». Quelles sont, précisément, les circonstances qui amènent la viviparité chez le sarcopte de la gale ? Nous 
n'avons pas pris connaissance d'aucun autre texte, antérieur ou ultérieur, qui mentionne cette possibilité. Au contraire, dans toutes les descriptions plus récentes du Sarcoptes scabiei, et nous citerons ici celle de M. Neveu-Lemaire (1938), celle de E. Brumpt (1949), celle de E. N. Pavlovski (1948), il n'est pas stipulé, chez le parasite de la gale humaine, d'autre multiplication que par des œufs qui accomplissent leur évolution dans le milieu extérieur. Selon E. Martini (1952) il se pourrait pourtant

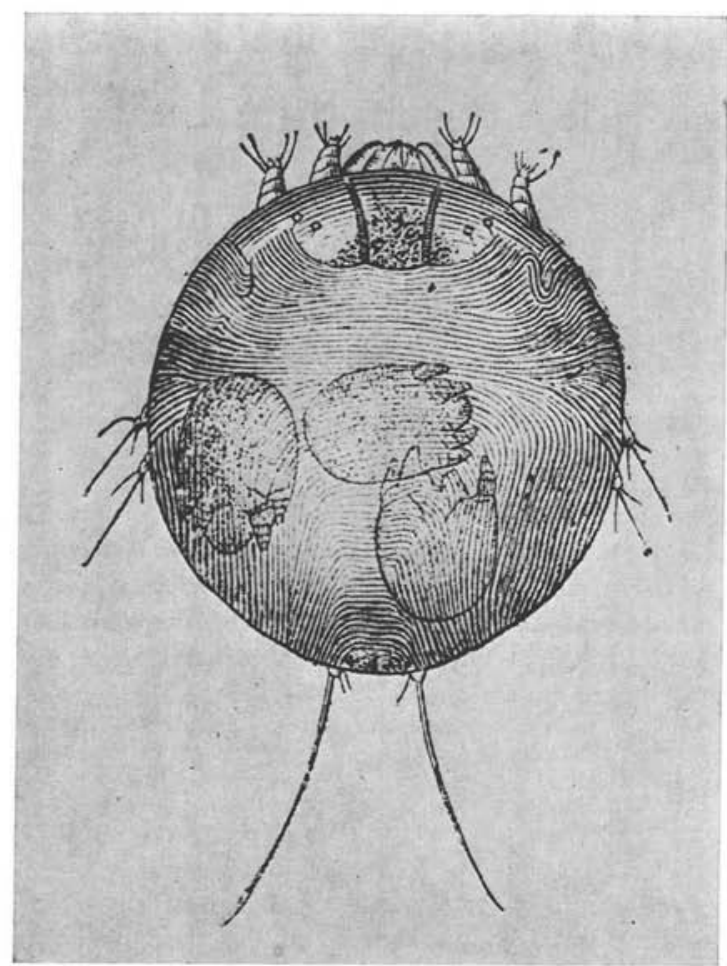

FIG. 1. - Cnemidocoptes laevis femelle vivipare (in A. Raillet)

que la femelle du sarcopte retienne plus que d'habitude les œufs dans son corps, ce qui permettrait le développement de la larve à l'intérieur de l'œuf avant que celui-ci soit déposé. Cette possibilité écourterait de beaucoup le temps nécessaire à l'éclosion de l'œuf dans le milieu extérieur.

Martini ajoute que selon Munro une telle possibilité n'arriverait pas dans des circonstances normales (page 313).

Quoi qu'il en soit, on ne peut pas parler de viviparité, même dans de telles conditions, mais toujours de ponte d'œufs, ces œufs possédant une larve plus ou moins formée à l'intérieur. 
Il existe toutefois, parmi les Acariens psoriques, des espèces chez lesquelles la viviparité représente un mode habituel de multiplication, comme dans le genre Cnemidocoptes. Nous reproduisons dans la figure 1 une femelle vivipare de Cnemidocoptes levis, représentée par A. Raillet dans son manuel cité ci-dessus.

Or, nous avons eu la chance de trouver parmi de nombreux sarcoptes provenant d'un cas de gale norvégienne, un exemplaire femelle qui présentait une larve

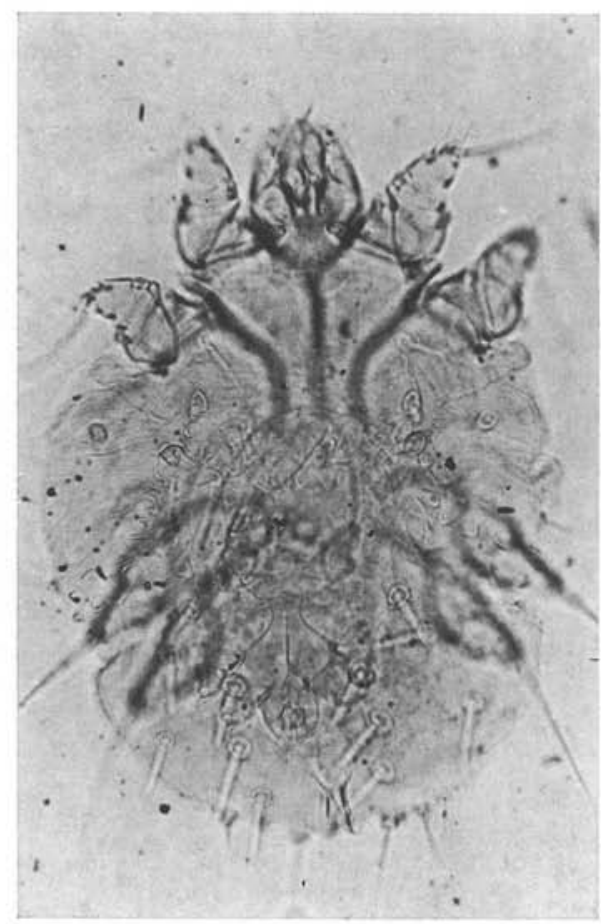

FIG. 2. - Sarcoptes scabili femelle devenue vivipare (original)

entièrement formée et libre dans son corps. Nous reproduisons, en microphotographie, dans la figure 2, cet exemplaire.

L'observation comparative des figures 1 et 2 montre clairement qu'il s'agit dans. les deux cas d'un même processus de viviparité.

L'exemplaire de sarcopte que nous présentons mesurait $0,320 \mathrm{~mm}$ en longueur sur $0,220 \mathrm{~mm}$ en largeur. Les épines du notogaster sont nettement bifurquées au sommet. Ces données concordent avec celles stipulées par de nombreux auteurs pour le sarcopte de la gale humaine commune (cf. A. H. Mandoul, 1925; A. Fialho, 1934). Nous sommes d'ailleurs convaincus, comme la plupart des auteurs, que la gale commune et la gale norvégienne reconnaissent un même agent étiologique 
et que la différence entre les manifestations cliniques des deux formes de la gale n'est due qu'au terrain différent offert par l'hôte au parasite.

Un argument des plus convaincants qui vient à l'appui de cette conception est le fait d'observation courante que les personnes qui attrapent la gale en soignant des malades atteints de gale norvégienne font la forme commune de la gale.

Tels ont été, selon Joyeux, les cas observés par Pozzo. Telle a été l'observation de W. Dubreuilh et Flye-Sainte-Marie relatée par A. H. Mandoul. De même, les personnes de l'entourage du malade, dont provenait le sarcopte vivipare présenté dans cette note ont contracté une gale commune (communication verbale du $\mathrm{P}^{\mathrm{r}} \mathrm{S}$. Longhin).

La gale norvégienne ne représente donc qu'un aspect particulier de l'interdépendance «parasite-hôte».

Le principal caractère imprimé au sarcopte par le terrain de beaucoup plus réceptif que d'habitude est la multiplication exagérée du parasite. Cette multiplication exagérée présume une fertilité exagérée et il est à se demander si la viviparité de l'exemplaire que nous présentons ne pourrait être considérée comme la limite extrême à laquelle mène cette fertilité exagérée.

Nous présentons ce sarcopte comme un nouvel exemple de la possibilité d'une mutilpication par viviparité chez le Sarcoptes scabiei, possibilité signalée par Bourguignon et mentionnée dans Raillet. Nous ne connaissons pas les circonstances dans lesquelles se sont produits le ou les cas observés par Bourguignon. Dans le cas présent, nous mettons cette viviparité exceptionnelle sur le compte de la fertilité exagérée engendrée par les conditions du terrain, dans la gale norvégienne.

\section{Bibliographie}

1. Brumpt (E.), 1949. - Précis de Parasitologie, $\mathrm{VI}^{\mathrm{e}}$ édit., Masson, Paris.

2. Fialho (A.), 1934. - Sur un cas de gale norvégienne. Annales de Parasitologie. Paris, XII, 6,472 .

3. Joyeux (Ch.), 1923. - Sur un cas de gale norvégienne en Afrique occidentale. Ann. Parasit. hum. et comp., I, 1923, 2, 167.

4. Mandoul (A. H.), 1925. - A propos de l'acare de la gale norvégienne. Ann. Parasit. hum. et comp., III, 4, 394.

5. Martini (E.), 1952. - Lehrbuch der medizinischen Entomologie, IV ${ }^{\circ}$ Aufl., Fischer Gustav, Jena.

6. Neveu-Lemaire (M.), 1938. - Traité d'Entomologie médicale et vétérinaire, Vigot, Paris.

7. Pavlovski (E. N.), 1948. - Rukovodstvo po Parazitologii tcheloveka. Tome II, MoskvaLéningrad.

8. Raillet (A.), 1895. - Zoologie médicale et agricole, Paris. 